\title{
DEFORMATION OF BRODY CURVES AND MEAN DIMENSION
}

\author{
MASAKI TSUKAMOTO*
}

\begin{abstract}
The main purpose of this paper is to show that ideas of deformation theory can be applied to "infinite dimensional geometry". We develop the deformation theory of Brody curves. Brody curve is a kind of holomorphic map from the complex plane to the projective space. Since the complex plane is not compact, the parameter space of the deformation can be infinite dimensional. As an application we prove a lower bound on the mean dimension of the space of Brody curves.
\end{abstract}

\section{INTRODUCTION}

1.1. Main results. Let $z=x+y \sqrt{-1}$ be the natural coordinate in the complex plane $\mathbb{C}$. For a holomorphic curve $f=\left[f_{0}: f_{1}: \cdots: f_{N}\right]: \mathbb{C} \rightarrow \mathbb{C} P^{N}$ with holomorphic functions $f_{0}, f_{1}: \cdots, f_{N}$, we define the pointwise norm $|d f| \geq 0$ (with respect to the Fubini-Study metric) by

$$
|d f|^{2}=\frac{1}{4 \pi} \Delta \log \left(\left|f_{0}\right|^{2}+\left|f_{1}\right|^{2}+\cdots+\left|f_{N}\right|^{2}\right) \quad\left(\Delta:=\frac{\partial^{2}}{\partial x^{2}}+\frac{\partial^{2}}{\partial y^{2}}\right) .
$$

We call $f$ a Brody curve if it satisfies $|d f| \leq 1$ (cf. Brody [2]). Let $\mathcal{M}\left(\mathbb{C} P^{N}\right)$ be the space of Brody curves in $\mathbb{C} P^{N}$ with the compact-open topology. Then $\mathcal{M}\left(\mathbb{C} P^{N}\right)$ becomes an infinite dimensional compact space and it admits a natural $\mathbb{C}$-action:

$$
(f(z), a) \mapsto f(z+a) \quad \text { for a Brody curve } f(z) \text { and } a \in \mathbb{C} .
$$

This paper studies the "mean dimension" $\operatorname{dim}\left(\mathcal{M}\left(\mathbb{C} P^{N}\right): \mathbb{C}\right)$. Mean dimension is a notion defined by Gromov [5] (see also Lindenstrauss-Weiss [8] and Lindenstrauss [7]). Mean dimension is a "dimension of an infinite dimensional space". Intuitively (the precise definition will be given in Section 2),

$$
\text { "dim }\left(\mathcal{M}\left(\mathbb{C} P^{N}\right): \mathbb{C}\right)=\operatorname{dim} \mathcal{M}\left(\mathbb{C} P^{N}\right) / \operatorname{vol}(\mathbb{C}) " .
$$

When we study the space of holomorphic maps from a compact Riemann surface, its (virtual) dimension can be derived from the deformation theory (and the index theorem).

Date: April 25, 2022.

2000 Mathematics Subject Classification. 32H30.

Key words and phrases. Brody curve, deformation theory, mean dimension, the Nevanlinna theory.

*Supported by Grant-in-Aid for JSPS Fellows (19.1530) from Japan Society for the Promotion of Science. 
The main purpose of this paper is to develop a new deformation theory which can be applied to the computation of $\operatorname{dim}\left(\mathcal{M}\left(\mathbb{C} P^{N}\right): \mathbb{C}\right)$.

For a Brody curve $f$ we define the Shimizu-Ahlfors characteristic function $T(r, f)$ by

$$
T(r, f):=\int_{1}^{r} \frac{d t}{t} \int_{|z|<t}|d f|^{2} d x d y \leq \pi r^{2} / 2 .
$$

We define the "mean energy" $e(f)$ (see Tsukamoto [12]) by

$$
e(f):=\limsup _{r \rightarrow \infty} \frac{2}{\pi r^{2}} T(r, f) \in[0,1] .
$$

Let $e\left(\mathbb{C} P^{N}\right)$ be the supremum of $e(f)$ over $f \in \mathcal{M}\left(\mathbb{C} P^{N}\right)$. From the definition we have $e\left(\mathbb{C} P^{N}\right) \in[0,1]$, but actually we can prove (see Tsukamoto [10, 12])

$$
0<e\left(\mathbb{C} P^{N}\right)<1 .
$$

We call $f \in \mathcal{M}\left(\mathbb{C} P^{N}\right)$ an elliptic Brody curve if there exists a lattice $\Lambda \subset \mathbb{C}$ such that $f(z+\lambda)=f(z)$ for all $z \in \mathbb{C}$ and $\lambda \in \Lambda$. If $f$ is a non-constant elliptic Brody curve, then $e(f)>0$. Let $e\left(\mathbb{C} P^{N}\right)_{\text {ell }}$ be the supremum of $e(f)$ over elliptic Brody curves $f$ in $\mathbb{C} P^{N}$. Obviously $0<e\left(\mathbb{C} P^{N}\right)_{\text {ell }} \leq e\left(\mathbb{C} P^{N}\right)$. Using the argument in Tsukamoto [10, Section 4], we can prove that $e\left(\mathbb{C} P^{N}\right)_{e l l}$ and $e\left(\mathbb{C} P^{N}\right)$ asymptotically become equal to 1 :

$$
\lim _{N \rightarrow \infty} e\left(\mathbb{C} P^{N}\right)_{\text {ell }}=\lim _{N \rightarrow \infty} e\left(\mathbb{C} P^{N}\right)=1 .
$$

Our main result on the mean dimension is the following inequality:

\section{Theorem 1.1.}

$$
2 e\left(\mathbb{C} P^{N}\right)_{\text {ell }}(N+1) \leq \operatorname{dim}\left(\mathcal{M}\left(\mathbb{C} P^{N}\right): \mathbb{C}\right) \leq 4 e\left(\mathbb{C} P^{N}\right) N .
$$

This theorem has the following two consequences:

\section{Theorem 1.2.}

$$
4 e\left(\mathbb{C} P^{1}\right)_{\text {ell }} \leq \operatorname{dim}\left(\mathcal{M}\left(\mathbb{C} P^{1}\right): \mathbb{C}\right) \leq 4 e\left(\mathbb{C} P^{1}\right) .
$$

\section{Theorem 1.3.}

$$
\left.2 \leq \liminf _{N \rightarrow \infty} \operatorname{dim}\left(\mathcal{M}\left(\mathbb{C} P^{N}\right): \mathbb{C}\right)\right) / N \leq \limsup _{N \rightarrow \infty} \operatorname{dim}\left(\mathcal{M}\left(\mathbb{C} P^{N}\right): \mathbb{C}\right) / N \leq 4 .
$$

Theorem 1.2 is the special case of Theorem 1.1. Theorem 1.3 comes from (3). The point of Theorem 1.3 is that the estimate is explicit. (The mean dimension $\operatorname{dim}\left(\mathcal{M}\left(\mathbb{C} P^{N}\right): \mathbb{C}\right.$ ) is a very transcendental object.)

Theorem 1.2 leads us to the following conjecture (actually a second main purpose of this paper is to propose this conjecture to the mathematical community):

\section{Conjecture 1.4.}

$$
e\left(\mathbb{C} P^{1}\right)_{\text {ell }}=e\left(\mathbb{C} P^{1}\right) .
$$


If this is true, then we get the following (index-theorem-like) result:

$$
\operatorname{dim}\left(\mathcal{M}\left(\mathbb{C} P^{1}\right): \mathbb{C}\right)=4 e\left(\mathbb{C} P^{1}\right)
$$

I think this formula is (if it is true) astonishing because the definitions of the left-hand-side and right-hand-side of (4) are very different. (Mean dimension is a topological quantity of the space, and mean energy is defined by using the energy distribution of Brody curves.) Note that Conjecture 1.4 itself is a purely function-theoretic problem. It does not contain a notion in the mean dimension theory.

The upper bound, $\operatorname{dim}\left(\mathcal{M}\left(\mathbb{C} P^{N}\right): \mathbb{C}\right) \leq 4 e\left(\mathbb{C} P^{N}\right) N$, in Theorem 1.1 is already proved in Tsukamoto [12, Theorem 1.4 and 1.5] by using the Nevanlinna theory 1 . The task of this paper is to prove the lower bound: $\operatorname{dim}\left(\mathcal{M}\left(\mathbb{C} P^{N}\right): \mathbb{C}\right) \geq 2 e\left(\mathbb{C} P^{N}\right)_{\text {ell }}(N+1)$. In order to prove this, we will develop a deformation theory of Brody curves. This deformation theory is a step toward the "infinite dimensional geometry": The parameter space of the deformation can be infinite dimensional. (But this is very natural because the space of Brody curves is an infinite dimensional space.)

A technical new feature of our deformation theory is the following: Usually we construct deformation theory within the framework of " $L^{2}$-theory" (or sometimes $L^{p}$-theory for $p<\infty)$. But (I think that) $L^{2}$-theory is not suitable for our purpose and it is better to construct the theory in the settings of " $L^{\infty}$-theory". (The fact that $L^{\infty}$ is suitable for the mean dimension theory is also suggested by [11]. In [11] it is shown that the mean dimension of the unit ball in $\ell^{p}(\Gamma)$ is zero, where $1 \leq p<\infty$ and $\Gamma$ is a finitely generated infinite amenable group.) But the analysis in the $L^{\infty}$-settings is more complicated than that of $L^{2}$, and it is the main technical task of the paper.

1.2. Remark on Conjecture 1.4. An elliptic function $f$ constructed below might be a good candidate for the function which attains the supremum of $e(f)$. Actually the following $f$ is an extremal function of the Bloch-constant-type problem solved in BonkEremenko [1]. Put

$$
e_{1}:=1 / \sqrt{2}, \quad e_{2}:=e^{2 \pi \sqrt{-1} / 3} / \sqrt{2}, \quad e_{3}:=e^{4 \pi \sqrt{-1} / 3} / \sqrt{2}, \quad e_{4}:=\infty .
$$

These four points become the vertices of a regular tetrahedron inscribed in the Riemann sphere $S^{2}=\mathbb{C} P^{1}$. Let $\omega_{1}$ be a positive real number (which will be fixed later) and set $\omega_{2}:=\omega_{1} \exp (\pi \sqrt{-1} / 3)$. Let $\Delta \subset \mathbb{C}$ be the regular triangle whose vertices are $0, \omega_{1}, \omega_{2}$ and, $\tilde{\Delta} \subset \mathbb{C} P^{1}$ the spherical regular triangle whose vertices are $e_{1}, e_{2}, e_{4}$. From the Riemann mapping theorem there exists an (unique) one-to-one holomorphic map $f: \Delta \rightarrow \tilde{\Delta}$ which sends $0, \omega_{1}, \omega_{2}$ to $e_{1}, e_{4}, e_{2}$ respectively. From the reflection principle, $f$ can be extended to an elliptic function whose period lattice is $\Lambda:=\mathbb{Z}\left(2 \omega_{1}\right) \oplus \mathbb{Z}\left(2 \omega_{2}\right) \subset \mathbb{C}$. The set of critical points of $f$ is $\mathbb{Z} \omega_{1}+\mathbb{Z} \omega_{2} \subset \mathbb{C}$, and the critical values are $e_{1}, e_{2}, e_{3}, e_{4}$. We have

\footnotetext{
${ }^{1}$ For the upper bound, see also Gromov [5, p. 396, (c)] and Tsukamoto [12, Remark 1.6].
} 
$\operatorname{deg}\left(f: \mathbb{C} / \Lambda \rightarrow \mathbb{C} P^{1}\right)=2 . f$ satisfies

$$
\left(f^{\prime}\right)^{2}=K\left(f-e_{1}\right)\left(f-e_{2}\right)\left(f-e_{3}\right)=K\left(f^{3}-1 / \sqrt{8}\right)
$$

for some positive constant $K . \omega_{1}$ can be derived from $K$ by

$$
\omega_{1}=\frac{1}{\sqrt{K}} \int_{1 / \sqrt{2}}^{\infty} \frac{d x}{\sqrt{x^{3}-1 / \sqrt{8}}}=\frac{2^{1 / 4}}{\sqrt{K}} \int_{1}^{\infty} \frac{d x}{\sqrt{x^{3}-1}} .
$$

The spherical derivative $|d f|(z)$ defined in (1) is given by

$$
|d f|^{2}=\frac{1}{\pi} \frac{\left|f^{\prime}\right|^{2}}{\left(1+|f|^{2}\right)^{2}}=\frac{K}{\pi} \frac{\left|f^{3}-1 / \sqrt{8}\right|}{\left(1+|f|^{2}\right)^{2}} .
$$

Some calculation shows

$$
\sup _{z \in \mathbb{C}} \frac{\left|z^{3}-1 / \sqrt{8}\right|}{\left(1+|z|^{2}\right)^{2}}=1 / \sqrt{8}
$$

Therefore

$$
\sup _{z \in \mathbb{C}}|d f|^{2}(z)=\frac{K}{\pi \sqrt{8}}
$$

We choose $\omega_{1}$ so that $K=\pi \sqrt{8}$. Then $\sup _{z \in \mathbb{C}}|d f|(z)=1$ and $f$ becomes an elliptic Brody curve. Since the volume of the fundamental domain of $\Lambda$ in $\mathbb{C}$ is given by $|\mathbb{C} / \Lambda|=2 \sqrt{3} \omega_{1}^{2}$, we have

$$
e(f)=\frac{2}{|\mathbb{C} / \Lambda|}=\frac{2 \pi}{\sqrt{3}}\left(\int_{1}^{\infty} \frac{d x}{\sqrt{x^{3}-1}}\right)^{-2}=0.6150198678198 \cdots .
$$

From Theorem 1.2,

$$
\operatorname{dim}\left(\mathcal{M}\left(\mathbb{C} P^{1}\right): \mathbb{C}\right) \geq \frac{8 \pi}{\sqrt{3}}\left(\int_{1}^{\infty} \frac{d x}{\sqrt{x^{3}-1}}\right)^{-2}=2.460079471279 \ldots
$$

This inequality might be an equality.

1.3. Remark on residual dimension. We want to remark about the "residual dimension" introduced by Gromov (see [5, p. 330 and p. 346]). This subsection is logically independent of the proof of Theorem 1.1, and readers can skip it. (But the idea of this subsection is implicitly used in Section 3.) Let $\Lambda \subset \mathbb{C}$ be a lattice and $\mathcal{M}\left(\mathbb{C} P^{N}\right)_{\Lambda}$ be the set of Brody curves $f$ satisfying $f(z+\lambda)=f(z)$ for all $\lambda \in \Lambda . \mathcal{M}\left(\mathbb{C} P^{N}\right)_{\Lambda}$ is the set of fixed-points of the natural action of $\Lambda$ on $\mathcal{M}\left(\mathbb{C} P^{N}\right)$. In other words, $\mathcal{M}\left(\mathbb{C} P^{N}\right)_{\Lambda}$ is the space of holomorphic maps $f: \mathbb{C} / \Lambda \rightarrow \mathbb{C} P^{N}$ satisfying $|d f| \leq 1$. The usual deformation theory gives (cf. Section 3)

$$
\frac{1}{|\mathbb{C} / \Lambda|} \operatorname{dim} \mathcal{M}\left(\mathbb{C} P^{N}\right)_{\Lambda} \leq 2(N+1) \sup _{f \in \mathcal{M}\left(\mathbb{C} P^{N}\right)_{\Lambda}} e(f) \leq 2 e\left(\mathbb{C} P^{N}\right)_{e l l}(N+1) .
$$

In particular, Theorem 1.1 gives

$$
\begin{aligned}
\operatorname{resdim}\left(\mathcal{M}\left(\mathbb{C} P^{N}\right):\{n \Lambda\}_{n \geq 1}\right) & :=\liminf _{n \rightarrow \infty} \frac{1}{|\mathbb{C} / n \Lambda|} \operatorname{dim} \mathcal{M}\left(\mathbb{C} P^{N}\right)_{n \Lambda}, \\
& \leq 2 e\left(\mathbb{C} P^{N}\right)_{\text {ell }}(N+1) \leq \operatorname{dim}\left(\mathcal{M}\left(\mathbb{C} P^{N}\right): \mathbb{C}\right) .
\end{aligned}
$$


Moreover some consideration shows

$$
\sup _{\Lambda \subset \mathbb{C}} \operatorname{resdim}\left(\mathcal{M}\left(\mathbb{C} P^{N}\right):\{n \Lambda\}_{n \geq 1}\right)=2 e\left(\mathbb{C} P^{N}\right)_{\text {ell }}(N+1),
$$

where $\Lambda$ runs over all lattices in $\mathbb{C}$.

Remark 1.5. In (15) the residual dimension is not bigger than the mean dimension. But in general residual dimension can be bigger than mean dimension; Consider the natural action of $\mathbb{Z}$ on $[0,1]^{\mathbb{Z}}$. For $n \geq 1$ let $F_{n} \subset[0,1 / n]^{\mathbb{Z}}$ be the set of fixed-points of the action of $n \mathbb{Z}$ on $[0,1 / n]^{\mathbb{Z}}$. Set $X:=\bigcup_{n \geq 1} F_{n} . X$ becomes a $\mathbb{Z}$-invariant closed set in $[0,1]^{\mathbb{Z}}$. Let $X_{n}(n \geq 1)$ be the set of fixed-points of the action of $n \mathbb{Z}$ on $X$. Since $F_{n} \subset X_{n}$, we have $\operatorname{dim} X_{n}=n$. Therefore, $\operatorname{resdim}\left(X:\{n \mathbb{Z}\}_{n \geq 1}\right):=\liminf _{n \rightarrow \infty} \operatorname{dim} X_{n} / n=1$. On the other hand, it is not difficult to see $\operatorname{dim}(X: \mathbb{Z})=0$.

1.4. Remark: twisted-elliptic Brody curves. For a Brody curve $f: \mathbb{C} \rightarrow \mathbb{C} P^{N}$, we call $f$ a twisted-elliptic Brody curve if there exist a lattice $\Lambda \subset \mathbb{C}$ and a homomorphism (of groups) $\phi: \Lambda \rightarrow P U(N+1)$ such that

$$
f(z+\lambda)=\phi(\lambda) f(z) \quad \text { for all } z \in \mathbb{C} \text { and } \lambda \in \Lambda .
$$

Note that the projective unitary group $P U(N+1)$ is the holomorphic-isometry group of $\mathbb{C} P^{N}$. Perhaps it might be able to apply the methods in this paper to twisted-elliptic Brody curves also. I think this is a natural generalization. But I don't know whether this improves the estimate of the mean dimension or not. So I don't study this case in this paper. If there is a reader who has an interest in this case, please pursue it.

1.5. Organization of the paper. In Section 2 we review the definition and basic properties of mean dimension. In Section 3 we prove Theorem 1.1, assuming an analytic result about the "deformation theory of Brody curves" proved in Section 5. Section 4 is a preparation for Section 5. In Section 5 we develop the deformation theory of Brody curves and complete the proof of Theorem 1.1. We give a remark about Gromov's conjecture on rational curves and mean dimension in Section 6 .

1.6. Acknowledgement. I wish to thank Professors Minoru Murata and Yoshio Tsutsumi. They gave me several helpful advices on elliptic partial differential equations. Especially I learned the basic idea of the proof of Proposition 4.2 from Professor Minoru Murata. I also wish to thank Professor Katsutoshi Yamanoi for various valuable discussions. He gave me an important suggestion about Conjecture 1.4.

\section{REVIEW OF MEAN DIMENSION}

We review the definitions of mean dimension. For the detail, see Gromov [5] or Lindenstrauss-Weiss [8]. Let $(X, d)$ be a compact metric space, $Y$ a topological space. For $\varepsilon>0$, a continuous map $f: X \rightarrow Y$ is called an $\varepsilon$-embedding if we have $\operatorname{Diam} f^{-1}(y) \leq \varepsilon$ 
for all $y \in Y$. Let $\operatorname{Widim}_{\varepsilon}(X, d)$ be the minimum number $n \geq 0$ such that there are an $n$-dimensional polyhedron $K$ and an $\varepsilon$-embedding from $X$ to $K$. The following is proved in Gromov [5, p. 333]. (This is a basic result for us. So we will give its proof in Appendix.)

Proposition 2.1. Let $(V,\|\cdot\|)$ be an $n$-dimensional normed linear space (over $\mathbb{R})$. Let $B \subset V$ be the closed ball of radius $r>0$ with the distance $d(x, y):=\|x-y\|$. Then

$$
\operatorname{Widim}_{\varepsilon}(B, d)=n \quad \text { for all } \varepsilon<r .
$$

Suppose the Lie group $\mathbb{C}$ continuously acts on the compact metric space $X$. For any positive number $R$, we define the distance $d_{R}(\cdot, \cdot)$ on $X$ by

$$
d_{R}(p, q):=\sup _{z \in \mathbb{C},|z| \leq R} d(z \cdot p, z \cdot q) \quad \text { for } p, q \in X .
$$

Set

$$
\operatorname{Widim}_{\varepsilon}(X: \mathbb{C}):=\lim _{R \rightarrow \infty} \frac{1}{\pi R^{2}} \operatorname{Widim}_{\varepsilon}\left(X, d_{R}\right) .
$$

This limit always exists (see Gromov [5, pp. 335-338] and Lindenstrauss-Weiss [8, Appendix]). We define the mean dimension $\operatorname{dim}(X: \mathbb{C})$ by setting

$$
\operatorname{dim}(X: \mathbb{C}):=\lim _{\varepsilon \rightarrow 0} \operatorname{Widim}_{\varepsilon}(X: \mathbb{C}) .
$$

$\operatorname{dim}(X: \mathbb{C})$ is a topological invariant, i.e., it does not depend on the given distance $d$.

Let $\Lambda=\mathbb{Z} \omega_{1} \oplus \mathbb{Z} \omega_{2} \subset \mathbb{C}$ be a lattice $\left(\omega_{1}, \omega_{2} \in \mathbb{C}\right)$. Then $\Lambda$ also acts on $X$ and we can define the mean dimension $\operatorname{dim}(X: \Lambda)$ as follows: For any positive integer $n$ we set

$$
\Omega_{n}:=\left\{x \omega_{1}+y \omega_{2} \in \Lambda \mid x, y \in \mathbb{Z}, 0 \leq x, y \leq n-1\right\}
$$

We define the distance $d_{\Omega_{n}}(\cdot, \cdot)$ on $X$ by

$$
d_{\Omega_{n}}(p, q):=\max _{z \in \Omega_{n}} d(z \cdot p, z \cdot q) \quad \text { for } p, q \in X .
$$

Set (the following limit always exists)

$$
\operatorname{Widim}_{\varepsilon}(X: \Lambda):=\lim _{n \rightarrow \infty} \frac{1}{n^{2}} \operatorname{Widim}_{\varepsilon}\left(X, d_{\Omega_{n}}\right) .
$$

We define the mean dimension $\operatorname{dim}(X: \Lambda)$ by

$$
\operatorname{dim}(X: \Lambda):=\lim _{\varepsilon \rightarrow 0} \operatorname{Widim}_{\varepsilon}(X: \Lambda) .
$$

The following gives the relation between $\operatorname{dim}(X: \mathbb{C})$ and $\operatorname{dim}(X: \Lambda)$. (This is given in Gromov [5, p.329] and Lindenstrauss-Weiss [8, Proposition 2.7]. For its proof, see also Tsukamoto [12, Proposition 4.5].)

\section{Proposition 2.2.}

$$
\operatorname{dim}(X: \Lambda)=|\mathbb{C} / \Lambda| \operatorname{dim}(X: \mathbb{C}),
$$

where $|\mathbb{C} / \Lambda|$ denotes the volume the fundamental domain of $\Lambda$ in $\mathbb{C}$. 


\section{Proof of Theorem 1.1}

Let $\Lambda \subset \mathbb{C}$ be a lattice and $\pi: \mathbb{C} \rightarrow \mathbb{C} / \Lambda$ be the natural projection. Let $\varphi: \mathbb{C} / \Lambda \rightarrow \mathbb{C} P^{N}$ be a non-constant holomorphic map satisfying $|d \varphi|<1$, and set $\tilde{\varphi}:=\varphi \circ \pi: \mathbb{C} \rightarrow \mathbb{C} P^{N}$. We have

$$
e(\tilde{\varphi})=\operatorname{deg} \varphi /|\mathbb{C} / \Lambda|,
$$

where $\operatorname{deg} \varphi=\left\langle c_{1}\left(\varphi^{*} \mathcal{O}(1)\right),[\mathbb{C} / \Lambda]\right\rangle$. Let $T^{\prime} \mathbb{C} P^{N}$ be the holomorphic tangent bundle of $\mathbb{C} P^{N}$ and consider its pull-back $E:=\tilde{\varphi}^{*} T^{\prime} \mathbb{C} P^{N}$ over $\mathbb{C}$. $E$ is equipped with the Hermitian metric induced by the Fubini-Study metric. We define a Banach space $V$ as the space of bounded holomorphic sections of $E$ with the sup-norm $\|\cdot\|_{\infty}$ :

(8) $V:=\left\{u: \mathbb{C} \rightarrow E \mid u\right.$ is a holomorphic section and satisfies $\left.\|u\|_{\infty}:=\sup _{z \in \mathbb{C}}|u(z)|<\infty\right\}$.

The following result is the keystone of the proof of Theorem 1.1 .

Proposition 3.1. There are positive numbers $\delta$ and $C$ such that for any $u \in V$ with $\|u\|_{\infty} \leq \delta$ there exists a Brody curve $f_{u}: \mathbb{C} \rightarrow \mathbb{C} P^{n}$ satisfying the following:

(i) $f_{0}=\tilde{\varphi}$.

(ii) The map $B_{\delta} \ni u \mapsto f_{u} \in \mathcal{M}\left(\mathbb{C} P^{N}\right)$ is $\Lambda$-equivariant. Here $B_{\delta}=\left\{u \in V \mid\|u\|_{\infty} \leq \delta\right\}$ and we have considered the natural $\Lambda$-action on $E$ and $V$.

(iii) For any $u, v \in V$ with $\|u\|_{\infty},\|v\|_{\infty} \leq \delta$, we have

$$
C^{-1}\|u-v\|_{\infty} \leq \sup _{z \in \mathbb{C}} \underline{d}\left(f_{u}(z), f_{v}(z)\right) \leq C\|u-v\|_{\infty},
$$

where $\underline{d}(\cdot, \cdot)$ denotes the distance on $\mathbb{C} P^{N}$ defined by the Fubini-Study metric.

We will prove this proposition in Section 5 by constructing a "deformation theory" of $\tilde{\varphi}$. (Each $f_{u}$ is a "small deformation" of $\tilde{\varphi}$.) Here we prove Theorem 1.1, assuming Proposition 3.1 .

Proof of Theorem 1.1. To begin with, we define the distance $d(\cdot, \cdot)$ on $\mathcal{M}\left(\mathbb{C} P^{N}\right)$ by

$$
d(f, g):=\sum_{n \geq 1} 2^{-n} \sup _{|z| \leq n} \underline{d}(f(z), g(z)) \quad \text { for } f, g \in \mathcal{M}\left(\mathbb{C} P^{N}\right) .
$$

Let $\Lambda=\mathbb{Z} \omega_{1} \oplus \mathbb{Z} \omega_{2} \subset \mathbb{C}$ be a lattice in $\mathbb{C}\left(\omega_{1}, \omega_{2} \in \mathbb{C}\right)$. For any positive integer $n$ we set

$$
K_{n}:=\left\{x \omega_{1}+y \omega_{2} \in \mathbb{C} \mid x, y \in \mathbb{R}, 0 \leq x, y \leq n\right\} .
$$

$K_{n}$ is a fundamental domain of $n \Lambda$ in $\mathbb{C}$. There is a positive constant $C_{1}=C_{1}(\Lambda)$ such that

$$
\sup _{z \in K_{1}} \underline{d}(f(z), g(z)) \leq C_{1} d(f, g) \quad \text { for } f, g \in \mathcal{M}\left(\mathbb{C} P^{N}\right) .
$$

Then for any $n>0$ we have

$$
\sup _{z \in K_{n}} \underline{d}(f(z), g(z)) \leq C_{1} d_{\Omega_{n}}(f, g) \quad \text { for } f, g \in \mathcal{M}\left(\mathbb{C} P^{N}\right),
$$

where $\Omega_{n}$ and $d_{\Omega_{n}}(\cdot, \cdot)$ are defined by (6) and (17). 
Let $\varphi: \mathbb{C} / \Lambda \rightarrow \mathbb{C} P^{N}$ be a non-constant holomorphic map satisfying $|d \varphi|<1$. We define $\tilde{\varphi}, E$ and $V$ as before. For any positive integer $n$, let $\pi_{n}: \mathbb{C} / n \Lambda \rightarrow \mathbb{C} / \Lambda$ be the natural $n^{2}$-fold covering map, and set $\varphi_{n}:=\varphi \circ \pi_{n}: \mathbb{C} / n \Lambda \rightarrow \mathbb{C} P^{N}$. Consider

$$
V_{n}:=H^{0}\left(\mathbb{C} / n \Lambda, \mathcal{O}\left(\varphi_{n}^{*} T^{\prime} \mathbb{C} P^{N}\right)\right) .
$$

$V_{n}$ is the space of holomorphic sections of $\varphi_{n}^{*} T^{\prime} \mathbb{C} P^{N}$ over $\mathbb{C} / n \Lambda$, and it can be identified with the subspace of $V$ consisting of $n \Lambda$-invariant holomorphic sections of $E$. From the Riemann-Roch formula and the vanishing of $H^{1}$ (cf. Section 5), we have

$$
\operatorname{dim} V_{n}=2 \operatorname{dim}_{\mathbb{C}} V_{n}=2\left\langle\varphi_{n}^{*} c_{1}\left(\mathbb{C} P^{N}\right),[\mathbb{C} / n \Lambda]\right\rangle=2 n^{2}(N+1) \operatorname{deg} \varphi .
$$

(Actually we need only the inequality $\operatorname{dim} V_{n} \geq 2 n^{2}(N+1) \operatorname{deg} \varphi$ in this proof. Hence we don't need $H^{1}=0$.) Let $\delta, C$ be the positive constants in Proposition 3.1. Set $B_{\delta}\left(V_{n}\right):=\left\{u \in V_{n} \mid\|u\|_{\infty} \leq \delta\right\}$. For any $u \in B_{\delta}\left(V_{n}\right)$ there exists a Brody curve $f_{u}$. From the $\Lambda$-equivariance in Proposition 3.1 (ii), $f_{u}$ is $n \Lambda$-invariant (i.e., it can be considered as a holomorphic map from $\mathbb{C} / n \Lambda$ to $\mathbb{C} P^{N}$ ). Then from Proposition 3.1 (iii) and (10), for any $u, v \in B_{\delta}\left(V_{n}\right)$

$$
\|u-v\|_{\infty} \leq C \sup _{z \in \mathbb{C}} \underline{d}\left(f_{u}(z), f_{v}(z)\right)=C \sup _{z \in K_{n}} \underline{d}\left(f_{u}(z), f_{v}(z)\right) \leq C C_{1} d_{\Omega_{n}}\left(f_{u}, f_{v}\right) .
$$

Moreover Proposition 3.1 shows that the map $B_{\delta}\left(V_{n}\right) \rightarrow \mathcal{M}\left(\mathbb{C} P^{N}\right), u \mapsto f_{u}$, is continuous. Therefore for any $\varepsilon>0$

$$
\operatorname{Widim}_{\varepsilon}\left(\mathcal{M}\left(\mathbb{C} P^{N}\right), d_{\Omega_{n}}\right) \geq \operatorname{Widim}_{C C_{1} \varepsilon}\left(B_{\delta}\left(V_{n}\right),\|\cdot\|_{\infty}\right),
$$

where $B_{\delta}\left(V_{n}\right)$ is equipped with the distance $\|u-v\|_{\infty}$. Then Proposition 2.1 implies, for $\varepsilon<\delta / C C_{1}$,

$$
\operatorname{Widim}_{\varepsilon}\left(\mathcal{M}\left(\mathbb{C} P^{N}\right), d_{\Omega_{n}}\right) \geq \operatorname{dim} V_{n}=2 n^{2}(N+1) \operatorname{deg} \varphi .
$$

Note that $\delta / C C_{1}$ is independent of $n$ (this is the crucial point). Hence

$$
\operatorname{Widim}_{\varepsilon}\left(\mathcal{M}\left(\mathbb{C} P^{N}\right): \Lambda\right)=\lim _{n \rightarrow \infty} \frac{1}{n^{2}} \operatorname{Widim}\left(\mathcal{M}\left(\mathbb{C} P^{N}\right), d_{\Omega_{n}}\right) \geq 2(N+1) \operatorname{deg} \varphi
$$

for any $\varepsilon<\delta / C C_{1}$. Thus

$$
\operatorname{dim}\left(\mathcal{M}\left(\mathbb{C} P^{N}\right): \Lambda\right) \geq 2(N+1) \operatorname{deg} \varphi
$$

Using Proposition 2.2, we get

$$
\begin{aligned}
\operatorname{dim}\left(\mathcal{M}\left(\mathbb{C} P^{N}\right): \mathbb{C}\right) & =\frac{1}{|\mathbb{C} / \Lambda|} \operatorname{dim}\left(\mathcal{M}\left(\mathbb{C} P^{N}\right): \Lambda\right) \\
& \geq 2(N+1) \operatorname{deg} \varphi /|\mathbb{C} / \Lambda|=2(N+1) e(\tilde{\varphi}) .
\end{aligned}
$$

Then we can prove Theorem 1.1. Let $f \in \mathcal{M}\left(\mathbb{C} P^{N}\right)$ be any elliptic Brody curve. Take a positive number $c<1$ and set $g(z):=f(c z)$. Then $g$ is an elliptic Brody curve satisfying $|d g|<1$, and we can apply (11) to $g$ :

$$
\operatorname{dim}\left(\mathcal{M}\left(\mathbb{C} P^{N}\right): \mathbb{C}\right) \geq 2(N+1) e(g)=2 c^{2}(N+1) e(f) .
$$


Let $c \rightarrow 1$. Then

$$
\operatorname{dim}\left(\mathcal{M}\left(\mathbb{C} P^{N}\right): \mathbb{C}\right) \geq 2(N+1) e(f) .
$$

This shows Theorem 1.1.

Remark 3.2. In the above proof, each $B_{\delta}\left(V_{n}\right)$ describes a small deformation of $\varphi_{n}$ : $\mathbb{C} / n \Lambda \rightarrow \mathbb{C} P^{N}$. The small deformations of each $\varphi_{n}$ can be constructed by the usual deformation theory. The point of Proposition 3.1 is that we can construct the deformations of all $\varphi_{n}$ with the estimates independent of $n$; This is essential in the above proof.

\section{Analytic preliminaries}

This section is a preparation for the proof of Proposition 3.1 .

4.1. Helmholtz equation. We will need some elementary facts about the Helmholtz equation on the plane $\mathbb{R}^{2}$ :

$$
(-\Delta+\lambda) w=0, \quad \text { where } \lambda>0 \text { and } \Delta=\frac{\partial^{2}}{\partial x^{2}}+\frac{\partial^{2}}{\partial y^{2}} .
$$

Set

$$
w_{\lambda}(z):=\frac{1}{2 \pi} \int_{0}^{2 \pi} \exp \sqrt{\lambda}(x \cos \theta+y \sin \theta) d \theta .
$$

$w_{\lambda}$ satisfies (12) and $w_{\lambda}>0$. The following fact can be easily checked:

Lemma 4.1. The minimum value of $w_{\lambda}$ is $w_{\lambda}(0)=1$, and $w_{\lambda}(z) \rightarrow+\infty$ as $|z| \rightarrow \infty$.

4.2. $L^{\infty}$-estimate. Let $F$ be a holomorphic vector bundle over the complex plane $\mathbb{C}$ with a Hermitian metric $h$. Let $\bar{\partial}: \Omega^{0}(F) \rightarrow \Omega^{0,1}(F)$ be the Dolbeault operator, and $\nabla$ the canonical connection on $(F, h)$. We denote the formal adjoint of $\bar{\partial}$ and $\nabla$ by $\bar{\partial}^{*}$ and $\nabla^{*}$. We have the following Weintzenböck formula: for any $\xi \in \Omega^{0,1}(F)$

$$
\bar{\partial} \bar{\partial}^{*} \xi=\frac{1}{2} \nabla^{*} \nabla \xi+R \xi,
$$

where $R \xi=\left[\nabla_{\partial / \partial z}, \nabla_{\partial / \partial \bar{z}}\right] \xi$. Note that for $\xi=u \otimes d \bar{z}(u \in \Gamma(F))$ we have

$$
\nabla_{\partial / \partial z} \xi=\left(\nabla_{\partial / \partial z} u\right) \otimes d \bar{z}, \quad \nabla_{\partial / \partial \bar{z}} \xi=\left(\nabla_{\partial / \partial \bar{z}} u\right) \otimes d \bar{z} .
$$

For $\xi=u \otimes d \bar{z}$ and $\eta=v \otimes d \bar{z}(u, v \in \Gamma(F))$, we set $\langle\xi, \eta\rangle:=2 h(u, v)$. We suppose that $F$ is "positive" in the following sense: there exists a positive number $a$ such that for any $\xi \in \Omega^{0,1}(F)$

$$
\langle R \xi, \xi\rangle \geq a|\xi|^{2}
$$

Proposition 4.2. Let $\xi \in \Omega^{0,1}(F)$ be a $F$-valued $(0,1)$-form of class $\mathcal{C}^{2}$, and set $\eta:=\bar{\partial} \bar{\partial}^{*} \xi$. If $\|\xi\|_{\infty},\|\eta\|_{\infty}<\infty$, then

$$
\|\xi\|_{\infty} \leq \frac{8}{a}\|\eta\|_{\infty}
$$


Proof. There is a point $z_{0} \in \mathbb{C}$ satisfying $\left|\xi\left(z_{0}\right)\right| \geq\|\xi\|_{\infty} / 2$. We suppose $z_{0}=0$ for simplicity. We have

$$
\Delta|\xi|^{2}=-2 \operatorname{Re}\left\langle\nabla^{*} \nabla \xi, \xi\right\rangle+2|\nabla \xi|^{2} .
$$

Using the Weintzenböck formula (14) and $\eta=\bar{\partial} \bar{\partial}^{*} \xi$, we have

$$
\begin{aligned}
\Delta|\xi|^{2} & =-4 \operatorname{Re}\langle\eta, \xi\rangle+4\langle R \xi, \xi\rangle+2|\nabla \xi|^{2}, \\
& \geq-4 \operatorname{Re}\langle\eta, \xi\rangle+4 a|\xi|^{2} .
\end{aligned}
$$

Set $M:=4\|\xi\|_{\infty}\|\eta\|_{\infty}$. We have $(-\Delta+4 a)|\xi|^{2} \leq M$.

Set $w(z):=M w_{2 a}(z) / 2 a$, where $w_{2 a}$ is a function defined in (13). $w(z)$ satisfies

$$
(-\Delta+2 a) w=0, \quad w \geq M / 2 a .
$$

Then $(-\Delta+4 a) w=2 a w \geq M$. Therefore

$$
(-\Delta+4 a)\left(w-|\xi|^{2}\right) \geq 0
$$

Since $\|\xi\|_{\infty}<\infty$ and $w(z) \rightarrow \infty(|z| \rightarrow \infty)$, we have $w(z)-|\xi|^{2}>0(|z| \gg 0)$. Then we can apply the minimum principle (see Gilbarg-Trudinger [3, Chapter 3, Corollary 3.2]), and get

$$
w(0)-|\xi(0)|^{2} \geq 0
$$

Therefore

$$
\|\xi\|_{\infty}^{2} / 4 \leq|\xi(0)|^{2} \leq w(0)=M / 2 a=2\|\xi\|_{\infty}\|\eta\|_{\infty} / a
$$

Thus $\|\xi\|_{\infty} \leq 8\|\eta\|_{\infty} / a$.

4.3. Perturbation of a Hermitian metric. We briefly discuss a perturbation technique of a Hermitian metric. M. Gromov also discuss it in [5, p. 399]. Let $\Lambda \subset \mathbb{C}$ be a lattice and $\varphi: \mathbb{C} / \Lambda \rightarrow \mathbb{C} P^{N}$ a non-constant holomorphic map. Let $\varphi^{*} T^{\prime} \mathbb{C} P^{N} \rightarrow \mathbb{C} / \Lambda$ be the pullback of the holomorphic tangent bundle $T^{\prime} \mathbb{C} P^{N}$ with the Hermitian metric $h$ induced by the Fubini-Study metric. Since the holomorphic bisectional curvature of the Fubini-Study metric is positive, there is $c>0$ such that for any $u \in \Gamma\left(\varphi^{*} T^{\prime} \mathbb{C} P^{N}\right)$

$$
h(R u, u) \geq c|d \varphi|^{2}|u|^{2},
$$

where $R$ is the curvature defined by $R u:=\left[\nabla_{\partial / \partial z}, \nabla_{\partial / \partial \bar{z}}\right] u$ ( $\nabla$ is the canonical connection).

Lemma 4.3. There is a Hermitian metric $h^{\prime}$ on $\varphi^{*} T^{\prime} \mathbb{C} P^{N}$ satisfying the following: There exists $a>0$ such that for any $u \in \Gamma\left(\varphi^{*} T^{\prime} \mathbb{C} P^{N}\right)$

$$
h^{\prime}\left(R^{\prime} u, u\right) \geq a|u|^{2}
$$

where $R^{\prime}$ is the curvature of $h^{\prime}$. 
Proof. Set $h^{\prime}=e^{-f} h$ where $f$ is a real valued function defined later. Then for any $u \in \Gamma\left(\varphi^{*} T^{\prime} \mathbb{C} P^{N}\right)$

$$
R^{\prime} u=\frac{1}{4}(\Delta f) u+R u \quad \text { and } \quad h^{\prime}\left(R^{\prime} u, u\right)=e^{-f}\left\{\frac{1}{4}(\Delta f)|u|^{2}+h(R u, u)\right\} .
$$

Set $\{p \in \mathbb{C} / \Lambda \mid d \varphi(p)=0\}=:\left\{p_{1}, \cdots, p_{n}\right\}$. Let $\delta>0$ be a sufficiently small number and set $A:=\coprod_{i} B_{\delta}\left(p_{i}\right) \subset \mathbb{C} / \Lambda\left(B_{\delta}\left(p_{i}\right)\right.$ is the closed ball of radius $\delta$ centered at $\left.p_{i}\right)$. From (16) there is $c^{\prime}>0$ such that

$$
h(R u, u) \geq c^{\prime}|u|^{2} \quad \text { for } u \in\left(\varphi^{*} T^{\prime} \mathbb{C} P^{N}\right)_{p} \text { at } p \in A^{c}=(\mathbb{C} / \Lambda) \backslash A .
$$

Let $g$ be a real valued function on $\mathbb{C} / \Lambda$ satisfying

$$
\text { (i) } g>0 \text { on } A, \quad(i i) g \geq-c^{\prime} / 2 \text { on } A^{c}, \quad \text { (iii) } \int_{\mathbb{C} / \Lambda} g d x d y=0 .
$$

From the condition (iii), there exists $f$ satisfying $\Delta f / 4=g$. Therefore

$$
h^{\prime}\left(R^{\prime} u, u\right)=e^{-f}\left(g|u|^{2}+h(R u, u)\right) .
$$

From the conditions (i) and (ii), it is easy to see that there exists $a>0$ such that $h^{\prime}\left(R^{\prime} u, u\right) \geq a|u|^{2}$ for all sections $u$.

\section{Deformation theory}

In this section we prove Proposition 3.1 by constructing "deformation theory".

Remark 5.1. M. Gromov gives a certain "deformation" argument different from ours in [5, pp. 399-400].

5.1. Deformation and the proof of Proposition 3.1. Let $\Lambda \subset \mathbb{C}$ be a lattice and $\pi: \mathbb{C} \rightarrow \mathbb{C} / \Lambda$ the natural projection. Let $\varphi: \mathbb{C} / \Lambda \rightarrow \mathbb{C} P^{N}$ be a non-constant holomorphic map satisfying $|d \varphi|<1$ and set $\tilde{\varphi}:=\varphi \circ \pi$. Let $E:=\tilde{\varphi}^{*} T^{\prime} \mathbb{C} P^{N}$ be the pull-back of the holomorphic tangent bundle $T^{\prime} \mathbb{C} P^{N}$. $E$ is equipped with the Hermitian metric $h$ induced by the Fubini-Study metric. $E$ admits the natural $\Lambda$-action.

Let $k$ be a non-negative integer and $\alpha$ a real number satisfying $0<\alpha<1$. We want to define the Hölder spaces $\mathcal{C}^{k, \alpha}(E)$ and $\mathcal{C}^{k, \alpha}\left(\Omega^{0,1}(E)\right)$. Let $\left\{U_{n}\right\}_{n=1}^{m},\left\{U_{n}^{\prime}\right\}_{n=1}^{m}$ and $\left\{U_{n}^{\prime \prime}\right\}_{n=1}^{m}$ be open coverings of $\mathbb{C} / \Lambda$ satisfying the following (i), (ii), (iii).

(i) $\bar{U}_{n} \subset U_{n}^{\prime}$ and $\bar{U}_{n}^{\prime} \subset U_{n}^{\prime \prime}$, and all $U_{n}, U_{n}^{\prime}, U_{n}^{\prime \prime}$ are smooth regions i.e., their boundaries are smooth.

(ii) The covering map $\pi: \mathbb{C} \rightarrow \mathbb{C} / \Lambda$ can be trivialized on each $U_{n}^{\prime \prime}$, i.e., there is a disjoint union $\pi^{-1}\left(U_{n}^{\prime \prime}\right)=\coprod_{\lambda \in \Lambda} U_{n, \lambda}^{\prime \prime}$ such that each $U_{n, \lambda}^{\prime \prime}$ is a connected component of $\pi^{-1}\left(U_{n}^{\prime \prime}\right)$ and $\left.\pi\right|_{U_{n, \lambda}^{\prime \prime}}: U_{n, \lambda}^{\prime \prime} \rightarrow U_{n}^{\prime \prime}$ is biholomorphic. Set $U_{n, \lambda}:=\pi^{-1}\left(U_{n}\right) \cap U_{n, \lambda}^{\prime \prime}$ and $U_{n, \lambda}^{\prime}:=\pi^{-1}\left(U_{n}^{\prime}\right) \cap U_{n, \lambda}^{\prime \prime}$, then $\left.\pi\right|_{U_{n, \lambda}}: U_{n, \lambda} \rightarrow U_{n}$ and $\left.\pi\right|_{U_{n, \lambda}^{\prime}}: U_{n, \lambda}^{\prime} \rightarrow U_{n}^{\prime}$ are biholomorphic and we have disjoint unions $\pi^{-1}\left(U_{n}\right)=\coprod_{\lambda \in \Lambda} U_{n, \lambda}$ and $\pi^{-1}\left(U_{n}^{\prime}\right)=\coprod_{\lambda \in \Lambda} U_{n, \lambda}^{\prime}$. 
(iii) A bundle trivialization of $\varphi^{*} T^{\prime} \mathbb{C} P^{N}$ is given on each $U_{n}^{\prime \prime}$, i.e., we have a holomorphic bundle isomorphism $\left.\varphi^{*} T^{\prime} \mathbb{C} P^{N}\right|_{U_{n}^{\prime \prime}} \rightarrow U_{n}^{\prime \prime} \times \mathbb{C}^{N}$. Then we also have a trivialization of $E$ over each $U_{n, \lambda}^{\prime \prime}$ through the isomorphisms $U_{n, \lambda}^{\prime \prime} \rightarrow U_{n}^{\prime \prime}$.

Let $u$ be a section of $E$ (not necessarily holomorphic). From (iii) in the above, $\left.u\right|_{U_{n, \lambda}^{\prime \prime}}$ can be seen as a vector-valued function on $U_{n, \lambda}^{\prime \prime}$. Hence we can define its $\mathcal{C}^{k, \alpha}$-norm $\|u\|_{\mathcal{C}^{k, \alpha}\left(\bar{U}_{n, \lambda}\right)}$ over $\bar{U}_{n, \lambda}$ as a vector-valued function (see Gilbarg-Trudinger [3, Chapter 4]). We define the $\mathcal{C}^{k, \alpha}(E)$-norm of $u$ by

$$
\|u\|_{\mathcal{C}^{k, \alpha}(E)}:=\sup _{n, \lambda}\|u\|_{\mathcal{C}^{k, \alpha}\left(\bar{U}_{n, \lambda}\right)} .
$$

We define the Hölder space $\mathcal{C}^{k, \alpha}(E)$ as the space of sections of $E$ whose $\mathcal{C}^{k, \alpha}(E)$-norms are finite. For $\xi=u \otimes d \bar{z} \in \Omega^{0,1}(E)(u \in \Gamma(E))$ we define its $\mathcal{C}^{k, \alpha}\left(\Omega^{0,1}(E)\right)$-norm by

$$
\|\xi\|_{\mathcal{C}^{k, \alpha}\left(\Omega^{0,1}(E)\right)}:=\sqrt{2}\|u\|_{\mathcal{C}^{k, \alpha}(E)}
$$

and we define $\mathcal{C}^{k, \alpha}\left(\Omega^{0,1}(E)\right):=\mathcal{C}^{k, \alpha}(E) \otimes d \bar{z}$. Then $\mathcal{C}^{k, \alpha}(E)$ and $\mathcal{C}^{k, \alpha}\left(\Omega^{0,1}(E)\right)$ become Banach spaces. (In the above definition of the Hölder spaces we have not used the open sets $U_{n, \lambda}^{\prime}$. They will be used in the next subsection.)

The holomorphic tangent bundle $T^{\prime} \mathbb{C} P^{N}$ is the eigenspace of the complex structure $J$ on $T \mathbb{C} P^{N} \otimes_{\mathbb{R}} \mathbb{C}$ of eigenvalue $\sqrt{-1}$. We naturally identify $T^{\prime} \mathbb{C} P^{N}$ with the tangent bundle $T \mathbb{C} P^{N}$ by

$$
T \mathbb{C} P^{N} \ni u \longleftrightarrow u-\sqrt{-1} J u \in T^{\prime} \mathbb{C} P^{N}
$$

So $E$ can be identified with $\tilde{\varphi}^{*} T \mathbb{C} P^{N}$.

Consider (cf. McDuff-Salamon [9, Chapter 3])

$$
\Phi: \mathcal{C}^{1, \alpha}(E) \rightarrow \mathcal{C}^{0, \alpha}\left(\Omega^{0,1}(E)\right) \quad u \mapsto P_{u}(\bar{\partial} \exp u) \otimes d \bar{z}
$$

Here, exp : $T \mathbb{C} P^{N} \rightarrow \mathbb{C} P^{N}$ is the exponential map defined by the Fubini-Study metric, and $P_{u}: T_{\exp u} \mathbb{C} P^{N} \rightarrow T_{\tilde{\varphi}} \mathbb{C} P^{N}$ is the parallel transport along the geodesic exp tu $(0 \leq$ $t \leq 1) . \bar{\partial} \exp u \in T_{\exp u} \mathbb{C} P^{N}$ is defined by

$$
\bar{\partial} \exp u:=\frac{1}{2}\left(\frac{\partial}{\partial x} \exp u+J \frac{\partial}{\partial y} \exp u\right) .
$$

$\Phi$ is a smooth map between the Banach spaces, and it is $\Lambda$-equivariant. The map $\mathbb{C} \ni z \mapsto$ $\exp u(z) \in \mathbb{C} P^{N}$ becomes a holomorphic curve if and only if $\Phi(u)=0$. The derivative of $\Phi$ at the origin is the Dolbeault operator:

$$
(d \Phi)_{0}=\bar{\partial}: \mathcal{C}^{1, \alpha}(E) \rightarrow \mathcal{C}^{0, \alpha}\left(\Omega^{0,1}(E)\right)
$$

Proposition 5.2. The small deformation of $\tilde{\varphi}$ is unobstructed, i.e., there exists a $\Lambda$ equivariant bounded linear operator $Q: \mathcal{C}^{0, \alpha}\left(\Omega^{0,1}(E)\right) \rightarrow \mathcal{C}^{1, \alpha}(E)$ satisfying $\bar{\partial} \circ Q=1$. 
This proposition will be proved later. Let $V:=$ ker $\bar{\partial}$ be the kernel of (17)) (this definition coincides with (8) ). Note that $V$ is a complement of the image of $Q$ in $\mathcal{C}^{1, \alpha}(E)$ and that it is $\Lambda$-invariant. From the elliptic regularity (cf. Subsection 5.2), we have

$$
\|u\|_{\infty} \leq \text { const } \cdot\|u\|_{\mathcal{C}^{1, \alpha}(E)} \leq \text { const }^{\prime} \cdot\|u\|_{\infty} \quad \text { for any } u \in V
$$

where const and const ${ }^{\prime}$ are independent of $u$.

For $r>0$ set $B_{r}:=\left\{u \in V \mid\|u\|_{\infty} \leq r\right\}$. From Proposition 5.2 and the implicit function theorem, there are $\delta>0$ and a $\Lambda$-equivariant smooth map $g: B_{\delta} \rightarrow \operatorname{Image}(Q)$ satisfying

$$
\text { (i) } g(0)=0, \quad(i i) \Phi(u+g(u))=0 \text { for all } u \in B_{\delta}, \quad(i i i)(d g)_{0}=0 \text {. }
$$

Set $f_{u}:=\exp (u+g(u)): \mathbb{C} \rightarrow \mathbb{C} P^{N}$ for $u \in B_{\delta}$. We want to show that these $f_{u}$ satisfy the conditions in Proposition 3.1. From (i) and (ii) in (18), $f_{0}=\tilde{\varphi}$ and each $f_{u}$ is a holomorphic curve. Since $|d \varphi|<1$, if we choose $\delta$ sufficiently small, all $f_{u}\left(u \in B_{\delta}\right)$ become Brody curves, i.e., $\left|d f_{u}\right| \leq 1$. Since $g$ is $\Lambda$-equivariant, the map $B_{\delta} \ni u \mapsto f_{u} \in \mathcal{M}\left(\mathbb{C} P^{N}\right)$ is also $\Lambda$-equivariant.

If we choose $\delta>0$ sufficiently small, then there exists $K>0$ such that for all $u, v \in B_{\delta}$

$$
K^{-1}\|u+g(u)-v-g(v)\|_{\infty} \leq \sup _{z \in \mathbb{C}} \underline{d}\left(f_{u}(z), f_{v}(z)\right) \leq K\|u+g(u)-v-g(v)\|_{\infty}
$$

(this is a standard property of the exponential map) and we have

$$
\|g(u)-g(v)\|_{\infty} \leq \frac{1}{2}\|u-v\|_{\infty} .
$$

Here we have used the condition (iii) in (18). Hence

$$
\frac{1}{2} K^{-1}\|u-v\|_{\infty} \leq \sup _{z \in \mathbb{C}} \underline{d}\left(f_{u}(z), f_{v}(z)\right) \leq \frac{3}{2} K\|u-v\|_{\infty} .
$$

Then all the conditions in Proposition 3.1 have been proved (assuming Proposition 5.2).

5.2. Proof of Proposition 5.2. To begin with, we consider a perturbation of the Hermitian metric on $E$. $E$ has the Hermitian metric $h$ induced by the Fubini-Study metric. From Proposition 4.3, $\varphi^{*} T^{\prime} \mathbb{C} P^{N}$ admits a Hermitian metric which is "positive" in the sense of Proposition 4.3. Then, pulling back this metric to $E, E$ admits a $\Lambda$-invariant Hermitian metric $h^{\prime}$ satisfying (15) for some $a>0$. In this subsection we use this $h^{\prime}$ as the Hermitian metric on $E$. Note that the definitions of the Hölder spaces $\mathcal{C}^{k, \alpha}(E)$ and $\mathcal{C}^{k, \alpha}\left(\Omega^{0,1}(E)\right)$ does not use the Hermitian metric. So they are independent of the choice of the Hermitian metric. (The sup-norm $\|\cdot\|_{\infty}$ depends on the Hermitian metric, but the sup-norms defined by $h$ and $h^{\prime}$ are equivalent to each other.)

We prove Proposition 5.2 by showing that

$$
\bar{\partial} \bar{\partial}^{*}: \mathcal{C}^{2, \alpha}\left(\Omega^{0,1}(E)\right) \rightarrow \mathcal{C}^{0, \alpha}\left(\Omega^{0,1}(E)\right)
$$


is an isomorphism. (Note that the Dolbeault operator $\bar{\partial}$ is independent of the Hermitian metric $h^{\prime}$, but its formal adjoint $\bar{\partial}^{*}$ depends on $h^{\prime}$.) Then $Q:=\bar{\partial}^{*}\left(\bar{\partial} \bar{\partial}^{*}\right)^{-1}$ gives a $\Lambda$ equivariant right inverse of $\bar{\partial}$. The injectivity of (19) directly follows from the $L^{\infty}$-estimate in Proposition 4.2. So the problem is its surjectivity.

Lemma 5.3. If $\eta \in \mathcal{C}^{0, \alpha}\left(\Omega^{0,1}(E)\right)$ has a compact support, then there exists $\xi \in \mathcal{C}^{2, \alpha}\left(\Omega^{0,1}(E)\right)$ satisfying $\bar{\partial} \bar{\partial}^{*} \xi=\eta$.

Proof. We set

$$
L_{1}^{2}\left(\Omega^{0,1}(E)\right):=\left\{\xi \in L^{2}\left(\Omega^{0,1}(E)\right) \mid \nabla \xi \in L^{2}\right\},
$$

where $\nabla \xi$ is the distributional derivative of $\xi$. (The $L^{2}$-norm and the $L^{2}$-space are defined by using the Hermitian metric $h^{\prime}$.) Let $\xi \in \Omega^{0,1}(E)$ be a compact-supported smooth section. From the Weitzenböck formula (14),

$$
\begin{aligned}
\left\|\bar{\partial}^{*} \xi\right\|_{L^{2}}^{2} & =\left(\bar{\partial} \bar{\partial}^{*} \xi, \xi\right)_{L^{2}}=\left(\frac{1}{2} \nabla^{*} \nabla \xi+R \xi, \xi\right)_{L^{2}}, \\
& \geq \frac{1}{2}\|\nabla \xi\|_{L^{2}}^{2}+a\|\xi\|_{L^{2}}^{2} .
\end{aligned}
$$

Therefore for any $\xi \in L_{1}^{2}\left(\Omega^{0,1}(E)\right)$

$$
\left\|\bar{\partial}^{*} \xi\right\|_{L^{2}}^{2} \geq \frac{1}{2}\|\nabla \xi\|_{L^{2}}^{2}+a\|\xi\|_{L^{2}}^{2} .
$$

This means that the inner-product $\left(\bar{\partial}^{*} \xi_{1}, \bar{\partial}^{*} \xi_{2}\right)_{L^{2}}\left(\xi_{1}, \xi_{2} \in L_{1}^{2}\left(\Omega^{0,1}(E)\right)\right)$ is equivalent to the natural inner-product $\left(\xi_{1}, \xi_{2}\right)_{L_{1}^{2}}:=\left(\nabla \xi_{1}, \nabla \xi_{2}\right)_{L^{2}}+\left(\xi_{1}, \xi_{2}\right)_{L^{2}}$ on $L_{1}^{2}\left(\Omega^{0,1}(E)\right)$.

$\eta$ defines a bounded functional $(\cdot, \eta)_{L^{2}}: L_{1}^{2}\left(\Omega^{0,1}(E)\right) \rightarrow \mathbb{C}$. From the Riesz representation theorem, there (uniquely) exists $\xi \in L_{1}^{2}\left(\Omega^{0,1}(E)\right)$ satisfying $\left(\bar{\partial}^{*} \phi, \bar{\partial}^{*} \xi\right)_{L^{2}}=(\phi, \eta)_{L^{2}}$ for all $\phi \in L_{1}^{2}\left(\Omega^{0,1}(E)\right)$ and

$$
\|\xi\|_{L_{1}^{2}}:=(\xi, \xi)_{L_{1}^{2}}^{1 / 2} \leq \text { const } \cdot\|\eta\|_{L^{2}} .
$$

In particular, $\bar{\partial} \bar{\partial}^{*} \xi=\eta$ in the sense of distribution. (The above is a standard argument in the " $L^{2}$-theory".)

We want to show $\xi \in \mathcal{C}^{2, \alpha}\left(\Omega^{0,1}(E)\right)$. Remember the open covering $\mathbb{C}=\bigcup_{n, \lambda} U_{n, \lambda}=$ $\bigcup_{n, \lambda} U_{n, \lambda}^{\prime}=\bigcup_{n, \lambda} U_{n, \lambda}^{\prime \prime}(n=1, \cdots, m, \lambda \in \Lambda)$ used in the definition of the Hölder spaces. Each $\left.\xi\right|_{U_{n, \lambda}^{\prime \prime}}$ can be seen as a vector-valued function. From the Sobolev embedding $L_{2}^{2} \hookrightarrow$ $\mathcal{C}^{0}$, the elliptic regularity (see Gilbarg-Trudinger [3, Chapter 8]) and (20),

$$
\begin{aligned}
\left\|\left.\xi\right|_{U_{n, \lambda}}\right\|_{\infty} & \leq \text { const }_{n} \cdot\left\|\left.\xi\right|_{U_{n, \lambda}}\right\|_{L_{2}^{2}} \leq \operatorname{const}_{n}^{\prime}\left(\left\|\left.\xi\right|_{U_{n, \lambda}^{\prime}}\right\|_{L_{1}^{2}}+\left\|\left.\eta\right|_{U_{n, \lambda}^{\prime}}\right\|_{L^{2}}\right) \\
& \leq \operatorname{const}_{n}^{\prime \prime} \cdot\|\eta\|_{L^{2}},
\end{aligned}
$$

where const $_{n}$, const $_{n}^{\prime}$ and const ${ }_{n}^{\prime \prime}$ are positive constants which depend on $n=1, \cdots, m$. The point is that they are independent of $\lambda \in \Lambda$; this is due to the $\Lambda$-symmetry of the equation. Then

$$
\|\xi\|_{\infty} \leq \text { const } \cdot\|\eta\|_{L^{2}} \cdot
$$


From the Schauder interior estimate (see Gilbarg-Trudinger [3, Chapter 6]),

$$
\|\xi\|_{\mathcal{C}^{2, \alpha}\left(\bar{U}_{n, \lambda}\right)} \leq \operatorname{const}_{n}\left(\|\xi\|_{\infty}+\|\eta\|_{\mathcal{C}^{0, \alpha}\left(\bar{U}_{n, \lambda}^{\prime}\right)}\right) \leq \operatorname{const}\left(\|\eta\|_{L^{2}}+\|\eta\|_{\mathcal{C}^{0, \alpha}\left(\Omega^{0,1}(E)\right)}\right) .
$$

Here we have used the following fact (which can be easily checked):

$$
\sup _{n, \lambda}\|\eta\|_{\mathcal{C}^{0, \alpha}\left(\bar{U}_{n, \lambda}^{\prime}\right)} \leq \mathrm{const} \cdot\|\eta\|_{\mathcal{C}^{0, \alpha}\left(\Omega^{0,1}(E)\right)}\left(=\text { const } \cdot \sup _{n, \lambda}\|\eta\|_{\mathcal{C}^{0, \alpha}\left(\bar{U}_{n, \lambda}\right)}\right)
$$

Thus $\|\xi\|_{\mathcal{C}^{2, \alpha}\left(\Omega^{0,1}(E)\right)}<\infty$ and $\xi \in \mathcal{C}^{2, \alpha}\left(\Omega^{0,1}(E)\right)$.

Then we can prove that (19) is surjective (and hence isomorphic). Take an arbitrary $\eta \in \mathcal{C}^{0, \alpha}\left(\Omega^{0,1}(E)\right)$. Let $\phi_{k}(k \geq 1)$ be cut-off functions on the plane $\mathbb{C}$ such that $0 \leq \phi_{k} \leq 1$, $\phi_{k}(z)=1$ for $|z| \leq k$ and $\phi_{k}(z)=0$ for $|z| \geq k+1$. Set $\eta_{k}:=\phi_{k} \eta$. From Lemma 5.3, there exists $\xi_{k} \in \mathcal{C}^{2, \alpha}\left(\Omega^{0,1}(E)\right)$ satisfying $\bar{\partial} \bar{\partial}^{*} \xi_{k}=\eta_{k}$. From the $L^{\infty}$-estimate in Proposition 4.2,

$$
\left\|\xi_{k}\right\|_{\infty} \leq \text { const } \cdot\left\|\eta_{k}\right\|_{\infty} \leq \text { const } \cdot\|\eta\|_{\infty} .
$$

Using the Schauder interior estimate on each $U_{n, \lambda}^{\prime}$, we get

$$
\left\|\xi_{k}\right\|_{\mathcal{C}^{2, \alpha}\left(\bar{U}_{n, \lambda}\right)} \leq \operatorname{const}_{n}\left(\left\|\eta_{k}\right\|_{\mathcal{C}^{0, \alpha}\left(\bar{U}_{n, \lambda}^{\prime}\right)}+\left\|\xi_{k}\right\|_{\infty}\right) \leq \operatorname{const}_{n}^{\prime}\left(\left\|\eta_{k}\right\|_{\mathcal{C}^{0, \alpha}\left(\bar{U}_{n, \lambda}^{\prime}\right)}+\|\eta\|_{\infty}\right) .
$$

Since $\left.\eta_{k}\right|_{U_{n, \lambda}^{\prime}}=\left.\eta\right|_{U_{n, \lambda}^{\prime}}$ for $k \gg 1$ (for each fixed $(n, \lambda)$ ), $\left\{\left.\xi_{k}\right|_{U_{n, \lambda}}\right\}_{k \geq 1}$ is a bounded sequence in $\mathcal{C}^{2, \alpha}\left(\bar{U}_{n, \lambda}\right)$. Hence, if we choose a subsequence, $\left\{\left.\xi_{k}\right|_{U_{n, \lambda}}\right\}_{k \geq 1}$ becomes a convergent sequence in $\mathcal{C}^{2}\left(\bar{U}_{n, \lambda}\right)$ (by Arzela-Ascoli's theorem). Therefore (by using the diagonal argument) there exists $\xi \in \Omega^{0,1}(E)$ of class $\mathcal{C}^{2}$ such that $\left\{\left.\xi_{k}\right|_{U_{n, \lambda}}\right\}_{k \geq 1}$ converges to $\left.\xi\right|_{U_{n, \lambda}}$ in $\mathcal{C}^{2}\left(\bar{U}_{n, \lambda}\right)$ for each $(n, \lambda)$. Since $\bar{\partial} \bar{\partial}^{*} \xi_{k}=\eta_{k}$, we have $\bar{\partial} \bar{\partial}^{*} \xi=\eta$. From (22), $\|\xi\|_{\infty} \leq$ const $\cdot\|\eta\|_{\infty}<\infty$. Using the Schauder interior estimate, we get

$$
\|\xi\|_{\mathcal{C}^{2, \alpha}\left(\bar{U}_{n, \lambda}\right)} \leq \operatorname{const}_{n}\left(\|\eta\|_{\mathcal{C}^{0, \alpha}\left(\bar{U}_{n, \lambda}^{\prime}\right)}+\|\xi\|_{\infty}\right) \leq \operatorname{const}_{n}^{\prime}\left(\|\eta\|_{\mathcal{C}^{0, \alpha}\left(\bar{U}_{n, \lambda}^{\prime}\right)}+\|\eta\|_{\infty}\right) .
$$

From (21),

$$
\|\xi\|_{\mathcal{C}^{2, \alpha}\left(\Omega^{0,1}(E)\right)} \leq \text { const } \cdot\|\eta\|_{\mathcal{C}^{0, \alpha}\left(\Omega^{0,1}(E)\right)}<\infty .
$$

Therefore we get $\xi \in \mathcal{C}^{2, \alpha}\left(\Omega^{0,1}(E)\right)$ satisfying $\bar{\partial} \bar{\partial}^{*} \xi=\eta$. Then (19) is an isomorphism, and the proof of Proposition 5.2 is finished (and hence the proof of Theorem 1.1 is completed).

\section{Remark on Gromov's CONJECture on RAtional CURVES AND MEAN DIMENSION}

Gromov gives the following (very beautiful) conjecture in [5, p. 329].

Conjecture 6.1. Let $X \subset \mathbb{C} P^{N}$ be a projective manifold, and $\mathcal{M}(X)$ the space of Brody curves in $X$. Then $\operatorname{dim}(\mathcal{M}(X): \mathbb{C})>0$ if and only if $X$ contains a rational curve.

The "if" part is easy and the problem is the "only if" part. The purpose of this section is to show the following proposition: 
Proposition 6.2. There exists a compact Hermitian manifold $X$ such that $X$ contains no rational curve and satisfies $\operatorname{dim}(\mathcal{M}(X): \mathbb{C})>0$. Here $\mathcal{M}(X)$ is the space of holomorphic maps $f: \mathbb{C} \rightarrow X$ satisfying

$$
\sup _{z \in \mathbb{C}}|d f|(z):=\sup _{z \in \mathbb{C}} \sqrt{2}|d f(\partial / \partial z)| \leq 1 .
$$

This shows that the projectivity (or the Kähler condition) is essential in Conjecture 6.1. (Actually I feel that the following argument suggests that the true conjecture might be something like the following; if $\operatorname{dim}(\mathcal{M}(X): \mathbb{C})>0$ then there are "many" elliptic curves in $X$ (cf. Gromov [5, p. 330, EXAMPLE]).) We prove Proposition 6.2 by using an argument similar to that of Section 3, (But this case is much easier than the proof of Theorem 1.1; We don't need a serious analytic argument. In particular we don't use the results in Section 4,5. Perhaps we can also prove Proposition 6.2 by applying the argument in Gromov [5, pp. 385-388] to the following construction.)

The compact Hermitian manifold $X$ constructed below is actually known as a counterexample of "bend-and-break" technique for general complex manifolds (see Kollár-Mori [6. Example 1.8]). We follow the description of [6, Example 1.8].

Let $\mathbb{C} / \Lambda$ be an elliptic curve $(\Lambda$ is a lattice in $\mathbb{C}$ ). Let $L$ be a holomorphic line bundle of deg $\geq 2$ over $\mathbb{C} / \Lambda$ such that there exists two holomorphic sections $s, t$ of $L$ satisfying $\{z \in \mathbb{C} / \Lambda \mid s(z)=t(z)=0\}=\emptyset$. Set $F:=L \oplus L$. The vector bundle $F$ has the following four sections:

$$
(s, t), \quad(\sqrt{-1} s,-\sqrt{-1} t), \quad(t,-s), \quad(\sqrt{-1} t, \sqrt{-1} s) .
$$

These are $\mathbb{R}$-linearly independent all over $\mathbb{C} / \Lambda$. (Therefore $F$ becomes a product bundle as a real vector bundle.) Hence we can define a lattice bundle $\Gamma \subset F$ by

$$
\Gamma:=\left\{x_{1}(s, t)+x_{2}(\sqrt{-1} s,-\sqrt{-1} t)+x_{3}(t,-s)+x_{4}(\sqrt{-1} t, \sqrt{-1} s) \mid x_{1}, x_{2}, x_{3}, x_{4} \in \mathbb{Z}\right\} .
$$

We define a compact complex threefold $X$ by $X:=F / \Gamma$. (Topologically $X=T^{2} \times T^{4}=$ $T^{6}$.) Obviously $X$ contains no rational curve. But $X$ can contain lots of Brody curves as we will see below.

We give a Hermitian metric (of a complex vector bundle) to $F$ and a Hermitian metric (of a complex manifold) to $X$. Let $\pi: \mathbb{C} \rightarrow \mathbb{C} / \Lambda$ be the natural projection and $E:=\pi^{*} F$ the pull-back of $F$ by $\pi$. $E$ is equipped with the $\Lambda$-invariant Hermitian metric induced by the metric on $F$. Let $V$ be the (Banach) space of bounded holomorphic sections of $E$ with the sup-norm $\|\cdot\|_{\infty}$, and set $B_{\delta}(V):=\left\{u \in V \mid\|u\|_{\infty} \leq \delta\right\}$ for $\delta>0$. Let $p: F \rightarrow X$ be the natural projection. If we choose $\delta$ sufficiently small and consider some scale-change of the Hermitian metric of $X$, then, for any $u \in B_{\delta}(V), p \circ u: \mathbb{C} \rightarrow X$ belongs to $\mathcal{M}(X)$ and the map $\Phi: B_{\delta}(V) \ni u \mapsto p \circ u \in \mathcal{M}(X)$ becomes injective. (Here we consider $u \in B_{\delta}(V)$ as a map from $\mathbb{C}$ to $F$.) We define a distance $d(\cdot, \cdot)$ on $B_{\delta}(V)$ by

$$
d(u, v):=\sum_{n \geq 1} 2^{-n} \sup _{|z| \leq n}|u(z)-v(z)| \quad \text { for any } u, v \in B_{\delta}(V) .
$$


We consider the topology defined by this distance on $B_{\delta}(V)$. Then $B_{\delta}(V)$ becomes compact, and $\Phi: B_{\delta}(V) \rightarrow \mathcal{M}(X)$ becomes a $\Lambda$-equivariant continuous embedding (here we consider the compact-open topology on $\mathcal{M}(X))$. Hence $\operatorname{dim}(\mathcal{M}(X): \Lambda) \geq \operatorname{dim}\left(B_{\delta}(V)\right.$ : $\Lambda)$. Let $\pi_{n}: \mathbb{C} / n \Lambda \rightarrow \mathbb{C} / \Lambda$ be the natural $n^{2}$-fold covering. Then the argument in Section 3 shows

$$
\operatorname{dim}\left(B_{\delta}(V): \Lambda\right) \geq \lim _{n \rightarrow \infty} \frac{1}{n^{2}} \operatorname{dim} H^{0}\left(\mathbb{C} / n \Lambda: \mathcal{O}\left(\pi_{n}^{*} F\right)\right)=2 \operatorname{deg}(F)>0 .
$$

(This is an inequality of the type "mean-dimension $\geq$ residual-dimension".) Therefore

$$
\operatorname{dim}(\mathcal{M}(X): \mathbb{C})=\operatorname{dim}(\mathcal{M}(X): \Lambda) /|\mathbb{C} / \Lambda|>0
$$

Remark 6.3. The above $X$ does not admit a Kähler metric. In fact the space of holomorphic one-forms in $X$ is (complex) one-dimensional. Since the first Betti number of $X=T^{6}$ is 6 , the Hodge theory implies that there is no Kähler metric on $X$.

\section{Appendix A. Proof of Proposition 2.1}

Gromov [5, p. 333] proved Proposition 2.1 by using the notion "filling radius". (Filling radius is a notion introduced in his celebrated paper [4.) Our following proof is a variant of the argument of Lindenstrauss-Weiss [8, Lemma 3.2].

It is enough to prove that for the unit ball $B:=\{x \in V \mid\|x\| \leq 1\}$ we have

$$
\operatorname{Widim}_{\varepsilon}(B, d)=\operatorname{dim} V=n \quad \text { for } \varepsilon<1 \text {. }
$$

Suppose there exists $\varepsilon<1$ such that $\operatorname{Widim}_{\varepsilon}(B, d) \leq n-1$. Then there is a finite open covering $\left\{U_{i}\right\}_{i \in I}$ of $B$ such that $\delta:=\max _{i \in I} \operatorname{Diam} U_{i}<1$ and its order is $\leq n-1$, i.e., $U_{i_{1}} \cap U_{i_{2}} \cap \cdots \cap U_{i_{n+1}}=\emptyset$ for distinct $i_{1}, i_{2}, \cdots, i_{n+1} \in I$.

Let $\left\{\phi_{i}\right\}_{i \in I}$ be a partition of unity on $B$ satisfying supp $\phi_{i} \subset U_{i}$. Take an arbitrary point $p_{i}$ in $U_{i}$. We define a map $f: B \rightarrow B$ by $f(x):=-\sum_{i \in I} \phi_{i}(x) \cdot p_{i}$. For any $x \in B$ we have

$$
\|f(x)+x\|=\left\|\sum_{i \in I} \phi_{i}(x)\left(x-p_{i}\right)\right\| \leq \delta \sum_{i \in I} \phi_{i}=\delta .
$$

For each $x \in B$ we have $\sharp\left\{i \in I \mid \phi_{i}(x) \neq 0\right\} \leq n$. Therefore $f(B)$ is contained in a union of at most $n-1$ dimensional polyhedrons. In particular $f(B)$ does not contain an inner point. Hence there exists $a \in B$ such that $a \notin f(B)$ and $\|a\| \leq 1-\delta$. Then we can define $g: B \rightarrow \partial B$ by $g(x):=(f(x)-a) /\|f(x)-a\| . g$ does not have a fixed point. In fact if $g(x)=x$, then $x \in \partial B$ and $f(x)-a=x\|f(x)-a\|$. Then $f(x)+x-a=x(1+\|f(x)-a\|)$ and $\|f(x)+x-a\|=1+\|f(x)-a\|>1$. From (23),

$$
1<\|f(x)+x-a\| \leq\|f(x)+x\|+\|a\| \leq \delta+\|a\| \leq 1 .
$$

This is a contradiction. Therefore $g$ does not have a fixed point, and this contradicts the Brouwer fixed-point theorem. 


\section{REFERENCES}

[1] M. Bonk, A. Eremenko, Covering properties of meromorphic functions, negative curvature and spherical geometry, Ann. of Math. 152 (2000) 551-592

[2] R. Brody, Compact manifolds and hyperbolicity, Trans. Amer. Math. Soc. 235 (1978) 213-219

[3] D. Gilbarg, N. S. Trudinger, Elliptic partial differential equations of second order, Reprint of the 1998 edition, Classics in Mathematics, Springer-Verlag, Berlin (2001)

[4] M. Gromov, Filling Riemannian manifolds, J. Differential Geom. 18 (1983) 1-147

[5] M. Gromov, Topological invariants of dynamical systems and spaces of holomorphic maps: I, Math. Phys. Anal. Geom. 2 (1999) 323-415

[6] J. Kollár, S. Mori, Birational geometry of algebraic varieties, With the collaboration of C.H. Clemens and A. Corti, Cambridge Tracts in Mathematics, 134, Cambridge University Press, Cambridge (1998)

[7] E. Lindenstrauss, Mean dimension, small entropy factors and an embedding theorem, Inst. Hautes Études Sci. Publ. Math. 89 (1999) 227-262

[8] E. Lindenstrauss, B. Weiss, Mean topological dimension, Israel J. Math. 115 (2000) 1-24

[9] D. McDuff, D. Salamon, J-holomorphic curves and quantum cohomology, University Lecture Series, 6, American Mathematical Society, Providence (1994)

[10] M. Tsukamoto, A packing problem for holomorphic curves, preprint, arXiv: math.CV/0605353

[11] M. Tsukamoto, Mean dimension of the unit ball in $\ell^{p}$, preprint, http://www.math.kyoto-u.ac.jp/preprint/index.html, (2007)

[12] M. Tsukamoto, Moduli space of Brody curves, energy and mean dimension, preprint, arXiv:0706.2981

Masaki Tsukamoto

Department of Mathematics, Faculty of Science

Kyoto University

Kyoto 606-8502

Japan

E-mail address: tukamoto@math.kyoto-u.ac.jp 